\title{
Ambiguous Self-Induced Disinformation (ASID) Attacks: Weaponizing a Cognitive Deficiency
}

\author{
M. Canham ${ }^{1,2}$, S. Sütterlin ${ }^{3}$, T. F. Ask ${ }^{4,5}$, B. J. Knox ${ }^{4,5,6}$, L. Glenister. ${ }^{2}$, and R. G. Lugo ${ }^{4,5}$. \\ 1 Chief Executive Officer, Beyond Layer Seven, United States \\ E-mail: mcanham@belay7.com \\ 2 School of Modeling Simulation and Training, \\ University of Central Florida, Orlando, FL, USA \\ E-mail: mcanham@ist.ucf.edu
}

3 Faculty of Computer Science, Albstadt-Sigmaringen University, Germany

E-mail: suetterlin@hs-albsig.de

4 Department of Information Security and Communication Technology, Norwegian University of Science and Technology, Gjøvik, Norway

E-mail:torvalda@stud.ntnu.no; benjamin.j.knox@ntnu.no; ricardo.g.lugo@ntnu.no,

5 Faculty of Health, Welfare and Organisation, Østfold University College, Norway

6 Norwegian Armed Forces Cyber Defence, Norway

\begin{abstract}
Humans quickly and effortlessly impose narrative context onto ambiguous stimuli, as demonstrated through psychological projective testing and ambiguous figures. We suggest that this feature of human cognition may be weaponized as part of an information operation. Such Ambiguous Self-Induced Disinformation (ASID) attacks would employ the following elements: the introduction of a culturally consistent narrative, the presence of ambiguous stimuli, the motivation for hypervigilance, and a social network. ASID attacks represent a reduced-risk, lowinvestment on the part of the adversary with a potentially significant reward, making this a likely tactic of choice for information operators within the context of gray-zone conflicts.
\end{abstract}

Keywords: Disinformation, Cognitive Malware, Gray-Zone Conflict, Ambiguous Self-Induced Disinformation Attacks

\section{Introduction}

The Twilight Zone episode Monsters Are Due on Maple Street opens with scenes from a typical American town set in the 1950's. A brief but strange orb appears in the sky, it flashes, and strange 
sounds emanate, then it disappears. The townspeople wonder and speculate about what they have witnessed. Shortly afterward, strange events occur; electronics stop working, cars begin starting themselves, house lights flash on and off. Rod Serling, the narrator, warns the audience that the monsters are coming to Maple Street. After losing electrical service, the neighborhood gathers to make plans for moving forward. One of the children, a science fiction fan, explains that these events are like those of every alien landing story that he has read about. He continues that the fictional space aliens sent reconnaissance scouts who "looked like humans but weren't" ahead of the main invasion. More anomalies occur, the townspeople become anxious and begin accusing each other of being space aliens in disguise. Every odd behavior that anyone exhibits becomes scrutinized for indications that someone is not human. Scrutinization leads to accusations, then escalates into physical attacks, and finally murder. The camera zooms out, two aliens come into view as they are watching the mayhem below. One alien says to the other:

Understand the procedure now?

Just stop a few of their machines... throw them into darkness for just a few hours... and then sit back and watch the pattern (emerge).

And the pattern is always the same?

With few variations.

All we need to do is sit back and watch... And let them destroy themselves.

Rod Serling's voice returns as the screen fades, "The tools of conquest do not necessarily come with bombs... there are weapons that are simply thoughts, attitudes, and prejudices... Prejudices can kill, suspicion can destroy."

The events in this Twilight Zone episode provide an introduction and context for this article. The occurrence of an ambiguous event (the flashing orb in the sky) without explanation is amplified when observers are unable to impose context onto the sight due to a lack of requisite knowledge. The provision of a coherent narrative about aliens landing and sending advance parties who appear to be human is coupled with opaque language such as the of use the word 'landing' rather than 'invasion'. Intended or not, the word 'landing' is itself ambiguous: a landing might be peaceful or could be the beginning of an attack, the implication is up to the interpretation of the observer. As more anomalous events occur, the townspeople increasingly interpret these as hostile indicators, even though there is no explicit indication that this is the case. Finally, we (the audience) learn that aliens are in fact behind the anomalous events, but that they have no intention of landing and committing hostilities, rather their intention is to inject ambiguous information into the system and let their targets destroy themselves.

In the quest for information superiority and psychological dominance to weaken a rival country, entity or alliance, factors that affect the flow and type of information play a significant role in the manner in which decisions are made. Adversarial power in information warfare often relies upon deception, agitation, ambiguity and propagation of narratives to influence people into making decisions adverse to their own interest, and that favor the deceiver or manipulator conducting the information or influence operation (Bagge, 2019). Building on the fictional introductory narrative, this article introduces a potential real-world event where injecting ambiguous information into the information stream, coupled with a culturally consistent narrative, and hypervigilance can weaponize a normal feature of human cognition. The resultant disrupting effects are self-induced, concealing the asymmetrical [military] means. This attack categorically 
falls into the gray zone of conflict where approaches have renewed relevance due to technological advances and human cognitive vulnerabilities.

The article proceeds by presenting a theoretical and historical grounding for Ambiguous Self-Induced Disinformation (ASID) attacks. The key elements of ASID attacks are explained before the authors present examples and forecast potential arenas where ASID attack could be deployed.

\section{Imposing Order on a Chaotic World}

Human cognition quickly and effortlessly projects context onto ambiguous stimuli. We cognitively impose context so naturally that we need to make conscious and deliberate effort to ignore our own projections to truly perceive information as ambiguous. This cognitive imposition can be easily demonstrated by viewing ambiguous figures. In the figure below, the middle characters are perceived as either the capital letter ' $\mathrm{B}$ ' or the number ' 13 ' depending on the contextual information that is provided by the surrounding characters. In fact, the middle characters are identical and ambiguous. They are not in any way altered by the contextual characters, rather we impose the context on them through our background knowledge of the English alphabet and knowledge of numerals. An observer lacking this knowledge will see the same characters in both contexts because they lack the prerequisite knowledge required to impose this context. This occurs through a process referred to as knowledge-driven processing, also referred to as top-down processing. When we interpret the information in our environment, our subjective experience is that we are interpreting it without bias and simply taking in what is there through a data-driven process (Cavanagh, 1991; Goolkasian, 1987; Theeuwes, 2010; Kornmeier, \& Bach, 2012; Ishizu, 2013; Intaite et al, 2019). This data-driven processing, also referred to as bottom-up processing is how we perceive incoming information when we have no context for what we are perceiving. It is more common for us to process the stimuli we encounter through the lens of our prior knowledge, hence approach it from a top-down or knowledge-driven perspective, even when we subjectively feel as though we are interpreting it without bias (Cavanagh, 1991; Goolkasian, 1987; Theeuwes, 2010; Kornmeier, \& Bach, 2012; Ishizu, 2013; Intaite et al, 2019).

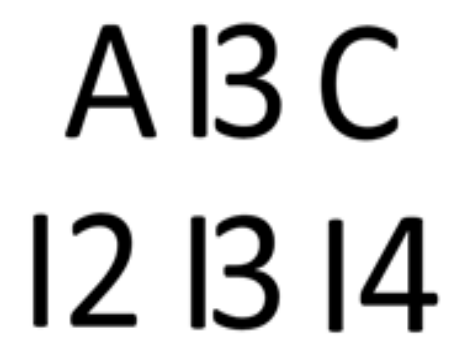

Figure 1: Ambiguous Figure

The presentation of ambiguous information that is also accompanied by a culturally consistent or plausible narrative (the latter serving as prior knowledge), can then lead to a form of self-induced disinformation effect which is potentially amplified by confirmation bias. In the figure above, the letter B or number 13 is perceived, depending on the context, but in fact this figure was created by combining the capital letter I and the number 3 to create an ambiguous figure. However, even with this information it is difficult to 'unsee' the B or 13, and this example involves low-level perception 
and known ground truth. What happens when the truth is less concrete and the context more ambiguous?

For over 100 years, psychologists have relied upon projective testing to gain insights into the inner workings of the human mind. These tests are effective because patients project their own interpretation onto the ambiguous stimuli in the test. An example of projective testing can be found in the Thematic Apperception Test (Murray, 1943). This test employs illustrations of characters engaged in ambiguous situations to which a patient or test subject is then asked what has led to the depicted event, what is happening in the moment of depiction, what the characters are experiencing, and what the outcome is. These tests demonstrate humans' ability to apply fabricated context to ambiguous stimuli, and this effect has been demonstrated through seeing figures in ambiguous images (Cavanagh, 1991; Goolkasian, 1987; Kornmeier, \& Bach, 2012; Ishizu, 2013; Intaite et al, 2019), or recognizing trends in stock market fluctuations (Cootner, 1964; Fama, 1970; Malkiel, 2003).

This leads us to ask the question, what if this feature of human cognition, the capacity to project context onto ambiguous stimuli, could be weaponized as part of an information operation through the presentation of a culturally consistent narrative paired with ambiguous information?

\section{Ambiguous Self-Induced Disinformation (ASID) Attacks}

Cyber operators and threat intelligence analysts are bombarded with information from sensors in machines, on networks, and from cyberspace in general. Sifting through too much information poses as much of a challenge as suffering from insufficient information. A major obstacle to successfully detecting attacks is that much of the information to sift through is ambiguous; it might indicate an attack, or it might be innocuous, such as a user visiting an unauthorized website from their work computer. Previous research suggests that these ambiguous signals could be weaponized to suppress network defender responses to attacks (Sawyer et al, 2016). These prevalence denial attacks (PDA), also known as grey signal attacks, leverage the "artificial inflation of signal probability flooding a network with grey signals, purposely built to be flagged by algorithmic defense systems but easily identified as non-threats by human operators" (Sawyer et al, 2016, p 162). This signal flood raises the noise to signal ratio and thereby compromises operator accuracy by suppressing vigilance capability.

We propose that such effects can be enhanced with the introduction of a culturally consistent narrative that induces knowledge-driven cognitive processing within the target leading to selfinduced disinformation influence. Such Ambiguous Self-Induced Disinformation (ASID) attacks might be used by threat actors as a 'meta-attack' apart from, or in parallel with, other information operations. ASID attacks manipulate the target's understanding of ambiguous stimuli by providing a context through which to interpret those stimuli. The principles underlying ASID Attacks have been implicitly understood for some time but has not been explicitly recognized as the potent form of weaponized psychology. We believe these attacks have been, and will be, highly effective because humans are highly capable at imposing order on a chaotic environment. For example, imagine that an advanced adversary (APT-X) announces that they will begin attacking a specific target on a certain day, at a certain time. Armed with this information, network defenders will likely begin focusing more acutely on network traffic around the time of the announced attack. This enhanced focus will lead them to increase their interpretation of ambiguous network activity 
as potentially malicious, even if no malicious activity is occurring. This would enable a malicious actor to consume defender bandwidth and resources at almost no cost to the attacker. This is one example of ASID attack employment, but this tactic is not limited to a cyber defense context.

\section{Mass Psychogenic Illness (MPI) - An Introduction}

Nearly every known culture throughout history has experienced a mass psychogenic illness (MPI) event. These MPI events follow similar patterns with slight variations. An ambiguous event or environmental stimuli (sight, sound, smell) is encountered, followed shortly after by a reaction in an individual that triggers similar reactions in other individuals within a social network. An early example of MPI episodes can be in the St. Vitus Dance of the fourteenth and fifteenth centuries. These episodes involved contagious dancing that would sometimes last for weeks. Processions of dancers would often end at chapels dedicated to the saint (hence the name) with victims lying on the ground from exhaustion and frothing at the mouth (Baloh \& Bartholomew, 2020). MPI episodes such as this are typically divided into two categories: sensory and motor. The St. Vitus Dance epidemic was an example of a motor MPI. In the twentieth and twenty-first centuries, sensory MPI events seem to be more common and are often brought about during high stress periods and in response to ambiguous stimuli such as sights, sounds, and smells (Baloh \& Bartholomew, 2020). For example, in 1989, shortly after Russian authorities deployed an irritant to disperse a crowd, approximately 400 Georgian schoolgirls began experiencing abdominal pain, skin irritation, and burning eyes in response to hearing rumors that they had also been exposed to the gas. Approximately one year later, several thousand residents of Kosovo experienced hyperventilation, burning sensations, headaches, dizziness, dry mouth, and nausea shortly after rumors circulated of Albanian dispersing neurotoxins to poison the local populace; however, no attacks were ever documented or proven to have occurred (Baloh \& Bartholomew, 2020).

Humans tend to apply a positive test strategy (a confirmation bias) when evaluating whether a phenomenon or attribute is applicable to oneself (Cialdini, 2016). In applying this strategy, people search their memory for positive instances, examples of something occurring or existing, rather than negative instances or the lack of something occurring or existing. If asked, "have you experienced any of these symptoms" people will think about points in their past when they experienced these, not when they did not experience such symptoms. This means that the person asking the question may (purposely or not) direct the person being asked through a psychological chute. Such psychological chuting causes people to focus on instances of a phenomenon's occurrence and can alter their perception of likelihood and relevance of that phenomenon (Cialdini, 2016, Chapter 2). This suggests that the introduction of a narrative that accompanies a phenomenon is likely to cause some proportion of a given population to identify with that narrative and thus psychological chuting may very well induce or facilitate MPI episodes.

Throughout history, MPI episodes have included the following characteristics: 1. an ambiguous situation, event, or environmental stimuli, 2. a culturally consistent narrative, 3. stressful environment, 4. response from the affected group, 5. diffusion of condition across the social network.

1. Ambiguous Situation, Event, Stimuli: Across the globe, well over 7,000 cases of persons hearing a chronic humming sound which induces anxiety, irritation, and sometimes painful reactions in those who suffer from the malady (Baloh \& Bartholomew, 2020). Accused sources of the humming noise have included power lines, wind turbines, telecommunications towers, industry fans, radio waves, birds, insects, and other natural 
and artificial sources. Reported symptoms include: a vague vibrating sensation, headache, nose pressure, nosebleeds, ear pain, ear popping, chest tightness, insomnia, short-term memory loss, general unwellness, nausea, stabbing pains, and occasionally violent illness (Baloh \& Bartholomew, 2020). A Canadian study of sufferers of humming induced maladies found that complaints for symptoms occurred at the same prevalence of similar symptoms in the general population. The researchers concluded that sufferers were misattributing common symptoms to the noises rather than being affected by them (Baloh \& Bartholomew, 2020). When testing the hypothesis that expectation could alter the interpretation of low frequency noise, researchers informed subjects that such noises could either cause symptoms like those reported by the noise sufferers, or could cause pleasant sensations and have therapeutic effects, depending on the condition that they were randomly assigned to. The researchers found that the expectations of the research subjects influenced their interpretation of the sound (Crichton et al, 2014; Crichton et al, 2015; Baloh \& Bartholomew, 2020). These studies suggest that ambiguous stimuli (sound in this case) may be interpreted differently depending on the observer's expectations and cultural context. The intensity of perceived physical symptoms can be enhanced or reduced by providing a coherent narrative modifying expectations. By providing a combination of ambiguous but not noxious thermal stimuli, positive, negative or emotionally neutral narratives elicited corresponding degrees of physical pain, eliciting a labelling effect that has been termed 'predictive coding' (Bräscher, Sütterlin, Scheuren, Van den Bergh \& Witthöft, 2020a). In the context of perceived environmental intolerance, Bräscher and colleagues (2020b) elicited somatic symptoms after sham-exposure to what healthy participants believed to be electromagnetic fields caused by a (sham) Wi-Fi device. The same effect of so-called somatosensory illusions was elicited as predicted by the predictive processing theory of somatic symptom generation following narratively induced attribution patterns and symptom expectations (Wolters et al., 2021). One explanation as to why some individuals generate symptoms, whereas others do not, despite being exposed to the same ambiguous stimuli and explanatory narratives, seems to be at least in parts be explained by individual traits related to the cognitive processing of potentially threatening stimuli (Scheuren, Sütterlin, \& Anton, 2014).

2. Culturally Consistent Narrative: The 1938 War of the Worlds radio broadcast became infamous for the public reaction that it provoked. The broadcast told the story of a Martian invasion of Earth, portrayed as though it was being reported in real time through a news broadcast. This radio show played on cultural narratives that were popular at the time; that Mars might be inhabited, and that war was imminent in Europe and the Pacific. Large numbers of audience members who tuned into the program after the disclosure announcement, were unaware that this was in fact a radio play and not actual news flashes. People panicked and began responding to the invasion as if it were real. Some even attacked water towers, which mimicked the three-legged Martian vehicles. The narrative of visitation from other worlds is consistent with scientifically oriented western cultures, but in other cultures that are more magically oriented, demonic possession is more common, as it was in pre-scientific Europe and North America. Collective narratives provide opportunities for knowledge-driven processing. They allow for interpretation (perceiving meaningful patterns instead of chaos) of the status quo, allow for projections into the future (what-ifs) and thus provide the basis for perceived control, resulting in stress alleviation (storytelling and narrative transportation). The stress alleviating effects of individual narratives are well established (Petko et al., 2015), and we argue that on a societal level, collective narratives can unfold a comparable effect of providing meaning to group members after the injection of ambiguous information into a social or sociotechnical system. The collective sensemaking following such a grey signal injection provides analogue to knowledge-based perceptual closure following the eradication of ambiguity. The collective narrative further maintains social bonds and group-level cooperation, which in turn contributes to reduced anxiety (see Bietti, Tilston, \& Bangerter, 2019). Collective narratives have an inherent self-stabilizing effect since adherence to it provides social proof to other individuals. The perceived state of the collective can also have 
measurable and potent biological effects on the individual. Perception of events related to the victory or defeat of a social group one considers oneself a member of (e.g., as a spectating supporter of a football team or political party) increases and decreases testosterone levels, respectively (Bernhardt et al., 1998; Stanton et al., 2009), more than most own behaviors. This exemplifies how mere perceptions related to the current state of the collective may biologically manifest in the individual in powerful ways that could have downstream influences on sensations such as pain (Hau et al., 2004), emotional regulation and cognitions (Celec et al., 2015; Martin et al., 2009), and behaviors (e.g., Dreher et al., 2016).

3. Stressful Environment: The majority, if not all, MPI events occur during times of stress for the affected group members. The 1990 MPI outbreak in Kosovo occurred at a time when rising ethnic tensions would eventually lead to the outbreak of civil war and Balkanization of the former Yugoslavia. The 1989 MPI episode in Georgia occurred amidst heightened tensions between Russia and Georgia during the Soviet Union disillusion. Even the earlier St. Vitus Dance MPI epidemics occurred on the foot heels of the Black Death devastation of Europe (Baloh \& Bartholomew, 2020). The stressful tension of an era appears to provide the fertile ground from which an MPI might flourish. Both short-term and especially long-term stress can have several negative effects on cognitive information processing (Porcelli \& Delgado, 2017; Provenzano et al., 2019). This is mainly a consequence of the effect that acute and chronic stressors have on prefrontal cortical function (Maeng \& Shors, 2013; Snyder et al., 2015) which is partly resulting from stress-induced hormonal interactions between the hypothalamus and prefrontal cortices that promote executive dysfunction (Uribe-Mariño et al., 2016). Concomitantly, the salience and persistence of prior or current stressors could increase the perceived likelihood that future stressors will occur due to a readiness to interpret stimuli in a manner congruent with one's psychological state (Andersen, 1990; Andersen \& Schwartz, 1992). On a neurocognitive level, negative affect decreases the efficiency of executive processing in favor of increased efficiency in mood-congruent salience-processing of stimuli, meaning that the individual focus on stimuli or aspect of stimuli that corresponds with their existing negative mood (Provenzano et al., 2019). A stress-induced reduction in executive function that favors mood-congruent information processing, combined with negative expectations and a positive testing strategy may shape sensory experiences in ways that facilitate individual-level psychogenic illness through predictive coding. It has been shown that expectations shape the encoding of perceptual stimuli at very early stages in perceptual processing (e.g. Aitken et al., 2020; Panichello \& Turk-Browne, 2021) suggesting a mechanistic basis for how an interplay between biasing top-down mood-congruent processes and exposure to ambiguous stimuli can produce MPI events. Once an individual witnesses or starts to experience somatic symptoms, interpersonal stress-related processes such as co-rumination may promote vigilance for symptom onset in other individuals or even aid in the spreading, exacerbating, and maintenance of symptoms (Carlucci et al., 2018; Hankin et al., 2010; Schwartz-Mette \& Rose, 2012). Co-rumination processes may additionally facilitate the continued hypothalamic-prefrontal cortex interactions that promote executive dysfunction and mood-congruent processing as well as a sustained failure to adapt to the MPI-associated stressors (Gianferante et al., 2014; Uribe-Mariño et al., 2016). Together, the above neurocognitive and sociocognitive factors demonstrate how stressful environments can interact with human cognition to produce MPI events.

4. Response from the Affected Group: A common element also present in most MPI episodes is the appearance of an Index Case, the first case which sets the expectations of those who follow. In the mid-1960's several African countries experienced MPI outbreaks of 'Laughing Mania'. These outbreaks primarily afflicted young schoolgirls presenting uncontrollable laughing as well as episodes of screaming, twitching, trance states and weeping. The pattern of contagion was for one or more of the afflicted to begin laughing uncontrollably and for this to spread to others. Other villages became afflicted with the disorder after news 
of the illness spread. A common theme across different MPI outbreaks of the initial case trend setting the symptomology of the subsequent cases. As mentioned in the Canadian study of sound sufferers, knowledge of the expression of symptoms may lead subsequent afflictees to attend more closely to otherwise normal sensations and thus attribute these to the 'illness'. Curiously, this disorder did not seem to spread to adult populations, but remained primarily contained within school children, particularly schoolgirls, suggesting a social network component to the spread of MPI outbreaks.

5. Diffusion Across the Network: In the early 1930's several towns in rural Virginia reported a series of gas attacks committed by elusive culprits who conspicuously always evaded capture or even left physical evidence of their presence. The index case was a family outside Roanoke County, Virginia, who reported on December 23, 1933, that someone had attempted to inject poisonous gas into their family's residence. Police investigations failed to find a suspect nor identify any physical evidence to support the claim. After the story appeared in local newspapers, reports of similar incidents proliferated throughout the Virginia countryside. The reports of these incidents skyrocketed after reporting but seem to have been localized to areas where the news was reported. In MPI episodes involving school children, it is common for children to become afflicted but not the adults. Similar trends were observed with the Laughing Mania outbreaks in Africa. Suggesting once again that a social network component may contribute to the spread of MPI outbreaks.

Weaponizing the mechanics that drive MPI events can be categorized as a gray-zone tactic. Characterized by ambiguity, opacity of the actors involved, uncertainty concerning the relevant policy and legal frameworks to address this attack vector is representative of the nature and form of this contemporary conflict space and reflects the challenges facing the security community attempting to understand, deter and respond to it (Votel, 2015). The significance and novelty of ASID attacks is the injection of ambiguous information as the primary, or peripheral, attack vector. The effects potentially transcend the information and psychological warfare domains to induce self-inflicted physical harm in targets.

\section{Elements of an ASID Attack}

Examining the components of MPI outbreaks provides clues to the elements required to conduct a success ASID attack. MPIs involve some ambiguous stimuli, either environmental or tied to an event. This ambiguous stimulus begs for resolution (Cialdini, 2016). The next component, a stressful environment or circumstance encourage hypervigilance and thus individuals begin attending closely to the ambiguous stimuli. Individuals within the social network then notice responses from affected individuals in the form of physical sensations. These sensations are often commonly experienced within a healthy population but are ignored or dismissed under 'normal' circumstances. Finally, the psychogenic condition becomes diffused across the social network. Drawing from MPI events as a guide, we suggest the following elements are necessary to successfully launch an ASID attack.

Introduction of a Culturally Consistent Narrative: Injecting a plausible narrative into the communication stream of the target population constitutes the required trigger to cause that population to engage in a positive test strategy to hyper-focus on ambiguous stimuli and interpret these through the lens of the weaponized narrative. 
The Presence of Ambiguous Stimuli: The presence of ambiguous stimuli provides the circumstances that the attack(s) exploit to mire the target in the information noise of innocuous and irrelevant information. The search for positive instances will encourage the target to discover false positives that will consume time and resources.

Motivation for Hypervigilance: The target needs to have a reason to care about examining the ambiguous stimuli, without this motivation, there is no reason to direct focus toward the ambiguous information stream. This hypervigilance of the ambiguous stimuli can lead targets to sensemaking of their experiences which are formed through cognitive modelling and experience formations. Such weaponized attacks rely on cognitive processes that have been shown to be both implicit and self-perpetuating. Memory implanting has been vastly researched and shown that individuals can crate experiences or memories though suggestion (see Roediger \& McDermott, 1995; Frenda et al., 2011). The previously described factors alongside the accessibility of ambiguous information, as well as actual reports of symptoms found in the media would contribute to individuals incorrectly interpreting their experiences and creating false memories. These experiences are then shared, both verbally and non-verbally with others causing a social contagion that would 'infect' others. Studies have shown that individuals can create experience and memories that leads to group experiences and collective memories, where individuals that experienced negative valence created more collective negative experiences, and this effect is stronger in smaller groups (Choi et al, 2017).

Optional Social Network: ASID attacks may infect an individual or an entire social network, but when targeted against a social network the additional biases of social proof and confirmation bias are introduced and reinforced respectively. Most leading social media platforms employ algorithms that propagate content that increase engagement among its users including content that induce strong negative emotions. These algorithms are not subject to any governmental or judicial regulations that inhibit their efficiency, thus offering a cost-effective and built-in 'infrastructure' for ASID attack propagation that requires minimal effort or understanding on the side of the adversary.

\section{Potential Attack Examples}

The following scenarios illustrate how such an ASID attack might be deployed by an adversary to degrade or disrupt an organization's ability to function. Firstly though, the much-publicized Havana Syndrome cannot be ignored as an example of an - ongoing - event where the existence of mysterious and ambiguous information is shaping opinion and behavior (see; Baloh and Bartholomew, 2020; BBC, 2021). In the context of ASID attacks it is inconsequential if the Havana Syndrome is a MPI caused by the sound of mating insects, malicious microwaves, or a remote incapacitation weapon using new physical principles. ASID attacks require no smoking gun. If the conditions are set (i.e. the culturally consistent narrative, the presence of ambiguous stimuli, motivation for hypervigilance, and if necessary, the accelerator function of social networks) then the ambiguity and fear it spreads act as multiplier in the target group. Victims will complete the 'play' themselves, as the real-power mechanics driving MPI events are weaponized as a component of an information warfare campaign targeting human cognition. 
The Telegraphed Attack: An advanced adversary who has established credibility through prior attacks and reputation may be capable of disrupting the operations of a target, or cause to consume a significant number of resources, by merely threatening or telegraphing an attack. If their primary goal is the degradation of operational capability or distraction, then an ASID attack might be an effective option. In this scenario, an APT might announce that they plan to launch an attack within a prescribed timeframe. If they are successful, information security staff from that organization will likely be more vigilant than their typical baseline during around the period of the announced attack. If this is a large, distributed organization, then this attack might be enhanced by impersonating organizational members on social media. These impersonated individuals could post disinformation regarding false Indicators of Compromise (IoCs). To maximize effectiveness, these IoCs should describe ambiguous network traffic. We believe this tactic could be highly effective because the injection of cognitive malware through social media channels would unlikely be anticipated and discovered in time to prevent the damage already accomplished by inducing hypervigilance within some operators.

Social Media Attack: Social media has provided a pathway for ASID attacks to propagate with little effort. Hot button topics are constantly enflamed on social media which are exacerbated by misinformation and disinformation. An adversary could create culturally consistent content (stimuli) that is meant to mislead a hypervigilant population. The disinformation is amplified through social shares which spread the content to an exponentially expanding network of Social Media users. This type of attack could be used to change opinion of voters in contentious battle ground state during an election period. A plausible scenario might involve a threat actor bringing attention to an ambiguous statement by a candidate about a hot button topic which causes a whirlwind of shares across the Social Media platform. By recruiting and inducing collective false memory within the targeted population, this ASID attack might cause a significant waste of time and resources to counter the effect and could lead to loss of confidence in election results.

Hoax Security Warning: It is common for security departments to send security warning emails to employees to caution against malicious email campaigns they have observed or other recent scams. A malicious threat actor might leverage these warnings to launch an ASID attack against the members of an organization by impersonating the security department in an email advising against a malware campaign being perpetrated against the organization. This email could contain descriptions of anomalous computer operations that in fact are normal but would appear as potentially malicious. Internet hoaxes such as these have already been observed in the wild with claims that users should look for the presence of the 'SULFNBK.exe' within their Windows C: drive directory. If identified, that the presence of this file indicated that their machine had been compromised, and that users should delete it from their system. In fact, this file was part of the normal operation of the machine and deleting it could potentially cause a system malfunction (AOL.com, 2015). A recent study on college student responses to phishing emails found that rather than reporting malicious emails to the university security department, some students posted screen shots of emails to subreddit forums (Canham et al, in preparation). This presents another potential ASID attack vector, in which malicious actors could pose as students posting a legitimate email as a scam.

False Bio-Attack: A variation of the above scenario would be to impersonate someone from an organization with the claim that a biological pathogen was released within a facility and that 
several employees have become ill as a result. The symptoms should describe commonly experienced symptoms such as headaches, dizziness, fatigue, hyperventilation, dry mouth, and nausea. To increase effectiveness by inducing hypervigilance, the hoax would then suggest that anyone experiencing these symptoms should immediately quarantine themselves and contact their doctor as the symptoms often rapidly increase in severity. This quarantine recommendation would serve two purposes. First, it would degrade operational capability, and second, it would provide an observable signal to other employees which might lead to additional 'cases' as has been observed in historical MPI events. The inducement of hypervigilance will statistically guarantee (in a large enough organization) that some individuals will experience these symptoms and associate them with the hoax attack. A variation of this hoax might describe exposure to electromagnetic radiation as the cause with similar symptoms and recommended course of action.

\section{Conclusion}

The long and well-documented history of Mass Psychogenic Illness (MPI) outbreaks suggests the potential effectiveness of the injecting ambiguous information, with a coherent narrative into the information stream, as a means for causing disruption. This form of attack exploits normal cognitive functions to cause widespread disruption. Information warriors should be aware of the possibility of the employment of such tactics in gray-zone conflict scenarios as these attacks would reside below the threshold of overt military actions. For the actors and agencies who use time and energy to investigate, understand, resolve, explain and potentially respond to ASID attacks, they must recognize the strategic validity of this approach and its capability to change how the victim, and they themselves, think and behave. Efforts should be made to better understand the key factors necessary to successfully launch such attacks and how to mitigate or disrupt their effects.

\section{References}

Aitken, F., Turner, G. and Kok, P. (2020). Prior Expectations of Motion Direction Modulate Early Sensory Processing. Journal of Neuroscience, 40 (33), 6389-6397. https://doi.org/10.1523/JNEUROSCI.0537-20.2020

America Online (AOL.com). (2015) Sulfnbk.exe Hoax Virus information. Originally at https://help.aol.com/articles/sulfnbkexe-hoax-virus-information. Retrieved on September 23, 2021, from https://archive.is/GOnLX.

Andersen, S. M., \& Schwartz, A. H. (1992). Intolerance of ambiguity and depression: A cognitive vulnerability factor linked to hopelessness. Social Cognition, 10(3), 271-298. https://doi.org/10.1521/soco.1992.10.3.271

Anderson, S. M. (1990). The inevitability of future suffering: The role of depressive predictive certainty in depression. Social Cognition, 8(2), 203-228.

https://doi.org/10.1521/soco.1990.8.2.203

Bagge, D. P. (2019). Unmasking Maskirovka: Russia's Cyber Influence Operations. Defense Press. 
Baloh, R. W., \& Bartholomew, R. E. (2020). Havana Syndrome: Mass Psychogenic Illness and the Real Story Behind the Embassy Mystery and Hysteria. Springer Nature.

BBC, (2021). CIA removes Vienna boss' over Havana syndrome outbreak. Accessed from https://www.bbc.com/news/world-europe-58675144 (29 September 2021)

Bernhardt PC, Dabbs JM Jr, Fielden JA, Lutter CD. Testosterone changes during vicarious experiences of winning and losing among fans at sporting events. Physiol Behav. 1998 Aug;65(1):59-62. doi: 10.1016/s0031-9384(98)00147-4.

Bietti, L. M., Tilston, O., \& Bangerter, A. (2019). Storytelling as adaptive collective sensemaking. Topics in Cognitive Science, 11(4), 710-732.

Bräscher, A. K., Schulz, S. M., Van den Bergh, O., \& Witthöft, M. (2020b). Prospective study of nocebo effects related to symptoms of idiopathic environmental intolerance attributed to electromagnetic fields (IEI-EMF). Environmental Research, 190, 110019.

Bräscher, A. K., Sütterlin, S., Scheuren, R., Van den Bergh, O., \& Witthöft, M. (2020a). Somatic Symptom Perception from a Predictive Processing Perspective: An Empirical Test Using the Thermal Grill Illusion. Psychosomatic Medicine, 82(7), 708-714.

Carlucci, L., D'Ambrosio, I., Innamorati, M., Saggino, A., \& Balsamo, M. (2018). Corumination, anxiety, and maladaptive cognitive schemas: when friendship can hurt. Psychology research and behavior management, 11, 133-144. https://doi.org/10.2147/PRBM.S144907

Cavanagh, P. (1991). What's up in top-down processing. Representations of vision: Trends and tacit assumptions in vision research, 295-304.

Celec, P., Ostatníková, D., \& Hodosy, J. (2015). On the effects of testosterone on brain behavioral functions. Frontiers in neuroscience, 9, 12. https://doi.org/10.3389/fnins.2015.00012

Choi, H. Y., Kensinger, E. A., \& Rajaram, S. (2017). Mnemonic transmission, social contagion, and emergence of collective memory: Influence of emotional valence, group structure, and information distribution. Journal of Experimental Psychology: General, 146(9), 1247.

Cialdini, R. (2016). Pre-suasion: A revolutionary way to influence and persuade. Simon and Schuster.

Cootner, P. H. (1964). The random character of stock market prices. MIT Press. ISBN 978-0262-03009-0.

Crichton F, Dodd G, Schmid G, Gamble G, Cundy T, Petrie KJ. The power of positive and negative expectations to influence reported symptoms and mood during exposure to wind farm sound. Health Psychol. 2014;33(12):1588-92. 
Crichton F, Dodd G, Schmid G, Petrie KJ. Framing sound: using expectations to reduce environmental noise annoyance. Environ Res. 2015; 142:609-14

Dreher, J.-D., Dunne, S., Pazderska, A., Frodl, T., Nolan, J. J., \& O’Doherty, J. P. (2016). Testosterone causes pro- and antisocial behaviors. Proceedings of the National Academy of Sciences, 113(41), 11633-11638; DOI: 10.1073/pnas.1608085113

Fama, E. (1970). Efficient Capital Markets: A Review of Theory and Empirical Work. Journal of Finance, 25 (2), 383-417. doi:10.2307/2325486.

Frenda, S. J., Nichols, R. M., \& Loftus, E. F. (2011). Current issues and advances in misinformation research. Current Directions in Psychological Science, 20, 20-23.

Gianferante, D., Thoma, M. V., Hanlin, L., Chen, X., Breines, J. G., Zoccola, P. M., \& Rohleder, N. (2014). Post-stress rumination predicts HPA axis responses to repeated acute stress. Psychoneuroendocrinology, 49, 244-252. https://doi.org/10.1016/j.psyneuen.2014.07.021

Goolkasian, P. (1987). Ambiguous figures: Role of context and critical features. Journal of General Psychology, 114(3), 217-228.

Hankin, B. L., Stone, L., \& Wright, P. A. (2010). Corumination, interpersonal stress generation, and internalizing symptoms: accumulating effects and transactional influences in a multiwave study of adolescents. Development and psychopathology, 22(1), 217-235.

https://doi.org/10.1017/S0954579409990368

Hau M, Dominguez OA, Evrard HC. Testosterone reduces responsiveness to nociceptive stimuli in a wild bird.

Horm Behav. 2004 Aug;46(2):165-70. doi: 10.1016/j.yhbeh.2004.02.007.

Intaite, M., Georgescu, A. L., Noreika, V., von Saldern, M. A., Vogeley, K., \& Falter-Wagner,

C. M. (2019). Adults with Autism Spectrum Condition Have Atypical Perception of Ambiguous Figures When Bottom-Up and Top-Down Interactions Are Incongruous. Autism: The International Journal of Research and Practice, 23(5), 1133-1142.

Ishizu, T. (2013). Disambiguation of ambiguous figures in the brain. Frontiers in human neuroscience, $7,501$.

Kornmeier, J., \& Bach, M. (2012). Ambiguous figures-what happens in the brain when perception changes but not the stimulus. Frontiers in human neuroscience, 6, 51.

Maeng, L. Y. and Shors, T. J. (2013): The stressed female brain: neuronal activity in the prelimbic but not infralimbic region of the medial prefrontal cortex suppresses learning after acute stress. Front Neural Circuits. 198. 
Malkiel, B. G. (2003). The Efficient Market Hypothesis and Its Critics. Journal of Economic Perspectives, 17(1), 59-82.

Martin, D. M., Burns, N. R., \& Wittert, G. (2009). Free testosterone levels, attentional control, and processing speed performance in aging men. Neuropsychology, 23(2), 158-167. https://doi.org/10.1037/a0014182

Panichello, M. F., \& Turk-Browne, N. B. (2021). Behavioral and Neural Fusion of Expectation with Sensation. Journal of cognitive neuroscience, 33(5), 814-825.

https://doi.org/10.1162/jocn_a_01684

Petko, D., Egger, N., Schmitz, F., Totter, A., Hermann, T., \& Guttormsen, S. (2015). Coping through blogging: A review of studies on the potential benefits of weblogs for stress reduction. Cyberpsychology - Journal of Psychosocial Research on Cyberspace, 9(2).

Porcelli, A. J., \& Delgado, M. R. (2017). Stress and Decision Making: Effects on Valuation, Learning, and Risk-taking. Current opinion in behavioral sciences, 14, 33-39. https://doi.org/10.1016/j.cobeha.2016.11.015

Provenzano, J., Verduyn, P., Daniels, N., Fossati, P., \& Kuppens, P. (2019). Mood Congruency Effects are Mediated by Shifts in Salience and Central Executive Network Efficiency. Social Cognitive and Affective Neuroscience. doi:10.1093/scan/nsz065

Roediger, H. L., \& McDermott, K. B. (1995). Creating false memories: Remembering words not presented in lists. Journal of Experimental Psychology: Learning, Memory, and Cognition, 21, 803-814.

Sawyer, B. D., \& Hancock, P. A. (2018). Hacking the human: the prevalence paradox in cybersecurity. Human factors, 60(5), 597-609.

Sawyer, B. D., Finomore, V. S., Funke, G., Warm, J. S., Matthews, G, Hancock, P. A. (2016). Cyber vigilance: The human factor. American Intelligence Journal, 32(2), 157-165. Retrieved on October 3, 2021, from https://bendsawyer.com/wp-content/uploads/2016/10/Sawyer-et-al.-2016Cyber-Vigilance-The-Human-Factor.pdf

Scheuren, R., Sütterlin, S., \& Anton, F. (2014). Rumination and interoceptive accuracy predict the occurrence of the thermal grill illusion of pain. BMC Psychology, 2(1), 1-15.

Schwartz-Mette, R. A., \& Rose, A. J. (2012). Co-rumination mediates contagion of internalizing symptoms within youths' friendships. Developmental psychology, 48(5), 1355-1365.

https://doi.org/10.1037/a0027484

Snyder, K. P., Barry, M. and Valentino, R. J. (2015): Cognitive impact of social stress and coping strategy throughout development. Psychopharmacology (Berl) 1: 185-195. 
Stanton SJ, Beehner JC, Saini EK, Kuhn CM, Labar KS. Dominance, politics, and physiology: voters' testosterone changes on the night of the 2008 United States presidential election. PLoS One. 2009 Oct 21;4(10):e7543. doi: 10.1371/journal.pone.0007543.

Theeuwes, J. (2010). Top-down and bottom-up control of visual selection. Acta psychologica, 135(2), 77-99.

Uribe-Mariño, A., Gassen, N. C., Wiesbeck, M. F., Balsevich, G., Santarelli, S., Solfrank, B., ... Schmidt, M. V. (2016). Prefrontal cortex corticotropin-releasing factor receptor 1 conveys acute stress-induced executive dysfunction. Biological Psychiatry, 80(10), 743-753. doi: 10.1016/j.biopsych.2016.03

Votel, Joseph L. (2015). 'The Gray Zone', White Paper, USSOCOM, Tampa, Florida.

Wolters, C., Harzem, J., Witthöft, M., Gerlach, A. L., \& Pohl, A. (2021). Somatosensory Illusions Elicited by Sham Electromagnetic Field Exposure: Experimental Evidence for a Predictive Processing Account of Somatic Symptom Perception. Psychosomatic Medicine, 83(1), 94-100. 